\title{
Tratamento de HIV no ano de 2020, por região do Brasil, segundo o DATASUS
}

\author{
HIV treatment in the year 2020, by region of Brazil, according to DATASUS \\ Tratamiento del VIH en el año 2020, por región de Brasil, según DATASUS
}

Larissa Távore Silva

ORCID: https://orcid.org/0000-0002-5837-7324

União das Faculdades dos Grandes Lagos, Brasil

E-mail: larissatavoree@gmail.com

Thiago de Almeida Garcia

ORCID: https://orcid.org/0000-0001-8457-9168

Universidade Brasil, Brasil

E-mail: thiagogarciaa16@gmail.com

Bruno Cavenaghi Castanheira

ORCID: https://orcid.org/0000-0002-2213-8655

Universidade Brasil, Brasil

E-mail: brunocavenaghi5@gmail.com

Beatriz Barboza Garavaso

ORCID: https://orcid.org/0000-0002-6506-4713

União das Faculdades dos Grandes Lagos, Brasil

E-mail: beatriz_garavaso@hotmail.com

Gabriela Andrade Roversi

ORCID: https://orcid.org/0000-0002-0214-2198

União das Faculdades dos Grandes Lagos, Brasil

E-mail: gabi_roversi@ hotmail.com

Mayara de Almeida Hentz Della Corte

ORCID: https://orcid.org/0000-0002-5554-2339 Universidade Brasil, Brasi

E-mail: mayara_hentz@hotmail.com

Thaina Mercado da Cunha

ORCID: https://orcid.org/0000-0001-7188-8703

Universidade de Marília, Brasil

E-mail: falecomathaina@ hotmail.com

Cássia Sousa Ferreira

ORCID: https://orcid.org/0000-0003-0990-5968 Universidade Brasil, Brasil

E-mail: sousacassia96@gmail.com

Caroline Silveira Damasceno

ORCID: https://orcid.org/0000-0002-9858-6550 Universidade Brasil, Brasil

E-mail: csdcrefono@gmail.com

Letícia Martins Bertati

ORCID: https://orcid.org/0000-0003-3040-0147 Universidade Brasil, Brasil

E-mail: leticia-bertati6@hotmail.com

Marília Sant'Ana Sequini

ORCID: https://orcid.org/0000-0003-2570-4947 Universidade Brasil, Brasil

E-mail: mariliasequini@ hotmail.com

Bianca Veloso Ferraz de Oliveira Silva

ORCID: https://orcid.org/0000-0001-6555-3798

Faculdade da Saúde e Ecologia Humana, Brasil E-mail: bvelosof@gmail.com

Gabriella Gil Aguila Saraiva Leitao

ORCID: https://orcid.org/0000-0001-7812-524X Unifadra - Fundec, Brasil

E-mail: gabiaguila28@gmail.com

Elenberg Chaves de Paula

ORCID: https://orcid.org/0000-0003-4170-7726

União das Faculdades dos Grandes Lagos, Brasil

E-mail: elenbergde@hotmail.com

Elizabete Santos Melo

ORCID: https://orcid.org/0000-0002-7322-9370

Universidade Brasil, Brasil

E-mail: enfaelizabetemelo@gmail.com 


\begin{abstract}
Resumo
O objetivo do estudo é avaliar os tratamentos de HIV/AIDS, nas diversas regiões do Brasil no ano de 2020. Trata-se de uma pesquisa documental. Utilizaram-se os dados estatísticos, do banco de dados do Departamento de Informática do Sistema Único de Saúde (DATASUS), do período de janeiro a dezembro de 2020, utilizando os filtros procedimentos hospitalares do SUS, tratamento de HIV/AIDS e todas as regiões do Brasil. A região que apresentou maior número de tratamentos no ano de 2020 foi a região sudeste, correspondendo a um total de 1551 tratamentos, responsável por $35,14 \%$ do total. A região que apresentou menores números de tratamentos foi a região norte, exibindo um total de 160 do total de tratamentos em 2020. Conclui-se, que o tratamento de HIV/AIDS no ano de 2020, portanto, foi mais realizado na região sudeste, que pode ser justificado, não só pela maior prevalência, como também pela maior distribuição dos recursos de saúde nessa área de abrangência.
\end{abstract}

Palavras-chave: HIV; Síndrome de Imunodeficiência Adquirida; Epidemiologia.

\begin{abstract}
The aim of the study is to evaluate the treatments for HIV/AIDS in different regions of Brazil in the year 2020. It is documentary research. Statistical data were used from the database of the Information Technology Department of the Unified Health System (DATASUS), from January to December of 2020, using filters for SUS hospital procedures, HIV/AIDS treatment and all regions do Brazil. The region with the highest number of treatments in 2020 was the Southeast region, corresponding to a total of 1551 treatments, accounting for $35.14 \%$ of the total. The region that presented the lowest number of treatments was the north region, showing a total of 160 of the total treatments in 2020. It is concluded that the treatment of HIV/AIDS in 2020, therefore, was more carried out in the Southeast region, which can be justified not only by the higher prevalence, but also by the greater distribution of health resources in this coverage area.
\end{abstract}

Keywords: HIV; Acquired Immunodeficiency Syndrome; Epidemiology.

\title{
Resumen
}

El objetivo del estudio es evaluar los tratamientos para el VIH / SIDA en diferentes regiones de Brasil en el año 2020. Se trata de una investigación documental. Se utilizaron datos estadísticos de la base de datos del Departamento de Tecnología de la Información del Sistema Único de Salud (DATASUS), de enero a diciembre de 2020, utilizando filtros para procedimientos hospitalarios del SUS, tratamiento de VIH / SIDA y todas las regiones de Brasil. La región con mayor número de Los tratamientos en 2020 fue la región Sudeste, correspondiente a un total de 1551 tratamientos, que representan el $35,14 \%$ del total. La región que presentó el menor número de tratamientos fue la región norte, mostrando un total de 160 del total de tratamientos en 2020. Se concluye que el tratamiento del VIH / SIDA en 2020, por lo tanto, se realizó más en la región Sudeste, lo cual puede justificarse no solo por la mayor prevalencia, sino también por la mayor distribución de los recursos de salud en esta área de cobertura.

Palabras clave: VIH; Síndrome de inmunodeficiencia adquirida; Epidemiología.

\section{Introdução}

A epidemia de HIV/AIDS no Brasil ainda é considerada estável a nível nacional. Desde meados do ano de 2012, foi possível observar uma certa diminuição da taxa de detecção de HIV no país, decorrente da recomendação do tratamento para todos os casos diagnosticados da doença, independente do critério clínico utilizado, além de tratamento para todos os casos diagnosticados da doença, independentemente dos critérios clínicos e imunológicos. Em 2018, novecentas mil pessoas viviam com HIV no Brasil, das quais $85 \%$ estavam diagnosticadas e $81 \%$ estavam vinculadas ao serviço de saúde (Pinto et al., 2021).

A prevalência da infecção pelo HIV e as suas taxas de incidência ainda variam de forma substancial, sendo que os países em desenvolvimento são os mais afetados. Destes, destaca-se como pior cenário a África Subsaariana, onde encontramse cerca de vinte e dois milhões de pessoas afetadas, constituindo um imenso desafio para o desenvolvimento de estratégias de controle e combate da pandemia (Polejack \& Seidl, 2010).

A Síndrome da Imunodeficiência Adquirida (AIDS), é uma manifestação clínica avançada da doença causada pelo vírus da imunodeficiência humana (HIV). Ela consiste em um transtorno intracelular que afeta a imunidade celular, resultando em maior suscetibilidade à infecções por doenças oportunistas e neoplasias. Sua transmissão se dá por via sexual, embora haja outras formas de exposição, tais como via sanguínea e via vertical. O tratamento é pautado a partir da Lei $\mathrm{n}^{\circ}$ 9.313/96, a qual determina que o governo garanta a distribuição de medicamentos antirretrovirais no âmbito do Sistema Único de Saúde (SUS), sendo o Brasil o primeiro país em desenvolvimento a adotar uma política pública que oferta acesso à Terapia Antirretroviral. 
Sob esse aspecto, em relação à adesão ao tratamento, faz-se uso de autorrelato, no qual é possível monitorar a adesão, tanto em pesquisas como na atenção cotidiana em saúde. (Coutinho et al., 2018; “L9313,” 2021).

O tratamento das pessoas acometidas com HIV/AIDS pode provocar algumas reações psicológicas, tais como ansiedade e depressão, as quais requerem atenção especial. Os sintomas de ansiedade observados em portadores de HIV estão relacionados às incertezas quanto à evolução da doença, evolução clínica, medida da dor, sofrimento e deterioração física, tratamento e morte. Dessa maneira, tais situações podem ser caracterizadas como uma resposta a situações estressantes, que variam de acordo com a interpretação de cada pessoa e, por vezes, faz-se necessário o auxílio de uma equipe multidisciplinar e apoio psicológico (Calvetti et al., 2014). Já a depressão está associada a crenças negativas em relação à doença e sentimentos de desesperança que prejudicam a motivação do indivíduo em cuidar de sua própria saúde e minam a confiança em sua capacidade de lidar com as demandas do tratamento. O apoio social é de suma importância para as pessoas com doenças crônicas se adaptarem às suas condições, de modo que os fatores psicossociais - acarretados pelo preconceito e discriminação presentes ainda na sociedade - não interfiram na adesão ao tratamento (Calvetti et al., 2014).

Embora os primeiros tratamentos contra o HIV tenham surgido na década de 1980, ainda hoje considera-se um problema de saúde pública. Nos anos de 2007 até 2020 foram notificados pelo Sistema de Informação de Agravos de Notificação (SINAN) 342.459 casos de infecção pelo HIV no Brasil, sendo 44,4\% na região sudeste, 20\% na região sul, 19\% na região nordeste, 9\% na região norte e 7,6\% na região centro-oeste. Desde 1996, o Sistema Único de Saúde distribui gratuitamente os antirretrovirais e, desde 2013, cobre o tratamento de todas as pessoas convivendo com HIV, independente da carga viral - medida que evita a transmissão da doença. Atualmente, há 21 medicamentos para tal finalidade e, embora a medicação não cure a infecção por HIV, ela é capaz de controlar a replicação viral, o que permite que o sistema imunológico do indivíduo seja fortalecido e tenha a capacidade para combater infecções secundárias.

Dessa maneira, objetiva-se com esse estudo demonstrar a epidemiologia de tal patologia, para que possa ser estudado futuramente como está sendo a prevenção, promoção e tratamento em saúde. Além disso, é de extrema importância para elaboração de políticas públicas para tentar mitigar esse quantitativo nas estatísticas entre os estados brasileiros. Infelizmente, é uma doença que ainda assombra a sociedade atualmente.

\section{Metodologia}

O estudo realizado foi feito em duas etapas, sendo que a primeira consistiu em uma pesquisa documental descritiva, na qual utilizou-se os dados estatísticos do banco de dados do Departamento de Informática do Sistema Único de Saúde (DATASUS), no período de janeiro a dezembro de 2020, utilizando os filtros: procedimentos hospitalares do SUS; tratamento de HIV/AIDS e todas as regiões do Brasil. Tal pesquisa permitiu a verificação de determinada problemática (organização do DataSUS) de forma indireta por meio dos documentos que foram produzidos pelo Ministério da Saúde, responsáveis pelo repositório dos dados (Calado, 2015). Já a segunda, baseou-se em uma busca realizada nas bases de dados Scielo: Scientific Electronic Library Online e Google Acadêmico, na qual utilizou-se os descritores “Tratamento", “HIV” e “Brasil” e "Tratamento de HIV no Brasil", respectivamente. Foram encontrados 178 artigos no sciELO que, após aplicação do filtro "Brasil", reduziram-se a 86 artigos; já no Google Acadêmico, encontraram-se 10.600 artigos, após aplicação do filtro "ano 2020". Posteriormente, foram selecionados 20 artigos para realização desse estudo.

Os critérios de exclusão foram os artigos que frisavam outras questões que não o tratamento do vírus HIV/AIDS e os que mencionavam outros países além do Brasil. 


\section{Resultados e Discussão}

A região que apresentou maior número de tratamentos no ano de 2020 foi a região Sudeste, correspondendo a um total de 1551 tratamentos, responsável por 35,14\% do total. A região que apresentou menores números de tratamentos foi a região Norte, exibindo um total de 160 do total de tratamentos em 2020. As outras regiões intermediárias apresentaram uma taxa de 61,22\% do total de tratamentos, sendo 1401 na região Nordeste, 811 na região Sul e 490 na região Centro-Oeste (Da Saúde, 2020).

Para exemplificar os resultados encontrados, os autores elaboraram um gráfico apresentando os dados obtidos através da busca no DATASUS.

Figura 1: Pessoas acometidas com HIV/AIDS em Tratamento segundo Regiões do Brasil.

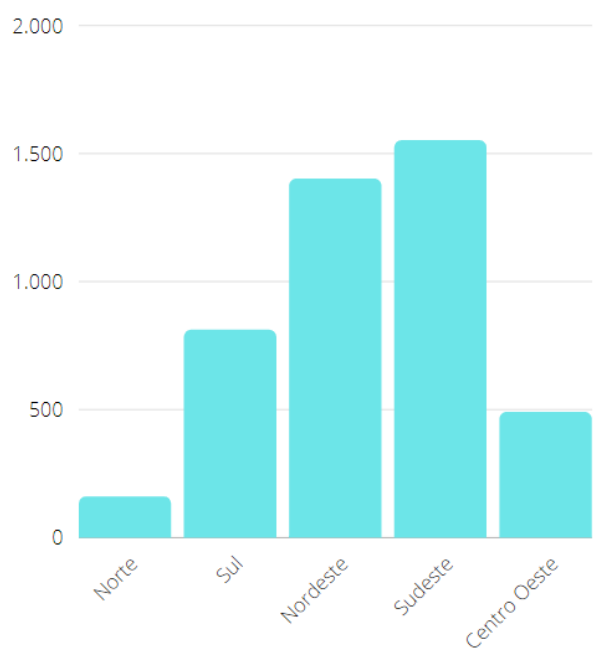

Fonte: Departamento de Informática do Sistema Único de Saúde (2020).

Quadro 1: Artigos selecionados.

\begin{tabular}{|c|c|c|c|}
\hline AUTORES E ANO & TÍTULO & OBJETIVOS & TIPO DE ESTUDO \\
\hline Batista et al., 2021 & $\begin{array}{l}\text { Prevalência de casos de HIV / AIDS nos } \\
\text { últimos } 10 \text { anos no Brasil }\end{array}$ & $\begin{array}{l}\text { Analisar, por meio de dados estatísticos ao longo dos } \\
\text { anos, qual a população mais afetada pela infecção } \\
\text { HIV/AIDS no Brasil, como o tratamento é administrado e } \\
\text { qual sua relação com as doenças oportunistas. }\end{array}$ & Estudo transversal \\
\hline Calvetti et al., 2014 & $\begin{array}{l}\text { Fatores psicossociais associados para } \\
\text { adesão ao tratamento e qualidade de vida } \\
\text { de pessoas que vivem com HIV/AIDS no } \\
\text { Brasil }\end{array}$ & $\begin{array}{l}\text { Investigar os fatores biopsicossociais que influenciam a } \\
\text { adesão ao tratamento e a qualidade de vida de indivíduos } \\
\text { que seguem com sucesso o tratamento anti-HIV / AIDS. }\end{array}$ & Estudo transversal \\
\hline Chaves et al., 2015 & $\begin{array}{l}\text { Estratégias para redução de preços de } \\
\text { medicamentos para HIV em situação de } \\
\text { monopólio no Brasil }\end{array}$ & $\begin{array}{l}\text { Analisar as estratégias governamentais para redução de } \\
\text { preço de medicamentos antirretrovirais para aids no } \\
\text { Brasil. }\end{array}$ & Análise quantitativa \\
\hline Costa et al., 2021 & $\begin{array}{l}\text { Fatores psicossociais envolvidos na adesão } \\
\text { ao tratamento do HIV/AIDS em adultos: } \\
\text { revisão integrativa da literatura }\end{array}$ & $\begin{array}{l}\text { Identificar na literatura os fatores psicossociais envolvidos } \\
\text { na adesão ao tratamento do HIV/AIDS em pacientes } \\
\text { adultos. }\end{array}$ & Revisão sistemática \\
\hline Coutinho et al., 2018 & $\begin{array}{l}\text { Tratamento antirretroviral: adesão e a } \\
\text { influência da depressão em usuários com } \\
\text { HIV/AIDS atendidos na atenção primária }\end{array}$ & $\begin{array}{l}\text { Identificar se a depressão interfere na adesão ao } \\
\text { tratamento antirretroviral. }\end{array}$ & $\begin{array}{c}\text { Estudo quantitativo e } \\
\text { qualitativo }\end{array}$ \\
\hline Fernandes et al., 2020 & $\begin{array}{l}\text { Tratamento do HIV/AIDS no Brasil: } \\
\text { impacto da adesão sobre a utilização de }\end{array}$ & $\begin{array}{l}\text { Determinar o impacto da adesão ao tratamento } \\
\text { antirretroviral sobre a utilização de recursos e custos }\end{array}$ & Revisão sistemática \\
\hline
\end{tabular}


Research, Society and Development, v. 11, n. 1, e6111124027, 2022

(CC BY 4.0) | ISSN 2525-3409 | DOI: http://dx.doi.org/10.33448/rsd-v11i1.24027

\begin{tabular}{|c|c|c|c|}
\hline & recursos e custos & relacionados ao manejo do HIV/AIDS no Brasil. & \\
\hline Gratão et al., 2021 & $\begin{array}{l}\text { Efeitos da HAART no estado nutricional } \\
\text { de crianças e adolescentes infectados pelo } \\
\text { HIV no Brasil: uma revisão sistemática }\end{array}$ & $\begin{array}{l}\text { Verificar os fatores associados ao crescimento, } \\
\text { composição corporal e aspectos alimentares e } \\
\text { nutricionais, após a infecção pelo HIV em crianças ou } \\
\text { adolescentes no Brasil. }\end{array}$ & Revisão sistemática \\
\hline Greco, 2016 & $\begin{array}{l}\text { Trinta anos de enfrentamento à epidemia } \\
\text { da AIDS no Brasil, 1985-2015 }\end{array}$ & $\begin{array}{l}\text { Descrever os êxitos e os indesculpáveis retrocessos destes } \\
30 \text { primeiros anos e as perspectivas para o controle e } \\
\text { eventual eliminação da aids. }\end{array}$ & Análise descritiva \\
\hline Martins, 2020 & $\begin{array}{l}\text { Adesão ao tratamento antirretroviral para o } \\
\text { HIV/AIDS: uma análise dos eventos } \\
\text { marcadores associados à adesão em um } \\
\text { estudo de coorte no município de } \\
\text { Alvorada-RS }\end{array}$ & $\begin{array}{l}\text { Analisar como eventos clínicos e sociais podem impactar } \\
\text { na adesão ao tratamento antirretroviral para o HIV. }\end{array}$ & Estudo coorte \\
\hline Mendicino et al., 2021 & $\begin{array}{l}\text { Recuperação imunológica após o início da } \\
\text { terapia antirretroviral: um desafio para } \\
\text { pessoas vivendo com HIV no Brasil }\end{array}$ & $\begin{array}{l}\text { Estimar o tempo até a recuperação imunológica e fatores } \\
\text { associados em pessoas vivendo com HIV (PVHIV) após } \\
\text { de iniciar a terapia antirretroviral (TARV). }\end{array}$ & Estudo coorte \\
\hline Monteiro et al., 2019 & $\begin{array}{l}\text { Desafios do tratamento como prevenção } \\
\text { do HIV no Brasil: uma análise a partir da } \\
\text { literatura sobre testagem }\end{array}$ & $\begin{array}{l}\text { Caracterizar os princípios e justificativas do TcP e discutir } \\
\text { os desafios da sua implementação. }\end{array}$ & Revisão sistemática \\
\hline Oliveira et al., 2020 & $\begin{array}{l}\text { Association between social support and } \\
\text { adherence to anti-retroviral treatment in } \\
\text { people living with HIV }\end{array}$ & $\begin{array}{l}\text { Investigar a associação entre suporte social, adesão à } \\
\text { terapia antirretroviral e fatores clínicos } \\
\text { sociodemográficos. }\end{array}$ & Estudo transversal \\
\hline Padoin et al., 2015 & $\begin{array}{l}\text { Mulheres do sul do Brasil em terapia } \\
\text { antirretroviral: perfil e o cotidiano } \\
\text { medicamentoso }\end{array}$ & $\begin{array}{l}\text { Descrever o perfil sociodemográfico, comportamental e } \\
\text { de tratamento de mulheres em terapia antirretroviral para } \\
\text { síndrome da imunodeficiência adquirida. }\end{array}$ & Estudo descritivo \\
\hline Passos \& Souza, 2015 & $\begin{array}{l}\text { Uma avaliação da qualidade de vida e seus } \\
\text { determinantes nas pessoas vivendo com } \\
\text { HIV/AIDS no sul do Brasil }\end{array}$ & $\begin{array}{l}\text { Avaliar a qualidade de vida e seus fatores associados em } \\
\text { pessoas vivendo com HIV/AIDS em um centro de } \\
\text { referência regional para o tratamento desta enfermidade } \\
\text { no Sul do Brasil. }\end{array}$ & Estudo transversal \\
\hline Pinheiro et al., 2020 & $\begin{array}{l}\text { O contexto sul-americano de divulgação } \\
\text { diagnóstica de adolescentes infectados } \\
\text { pelo HIV / AIDS: uma revisão sistemática } \\
\text { da literatura }\end{array}$ & $\begin{array}{l}\text { Analisar as evidências científicas sobre a divulgação do } \\
\text { diagnóstico de adolescentes infectados pelo HIV/AIDS no } \\
\text { contexto sul-americano. }\end{array}$ & Revisão sistemática \\
\hline Pinto et al., 2021 & $\begin{array}{l}\text { Protocolo brasileiro para Infecções } \\
\text { Sexualmente } \quad \text { Transmissíveis 2020: } \\
\text { infecção pelo HIV em adolescentes e } \\
\text { adultos }\end{array}$ & $\begin{array}{l}\text { Expor os sinais e sintomas da infecção pelo HIV e } \\
\text { realização de seu diagnóstico, a fim de oferecer } \\
\text { tratamento adequado e reduzir complicações. }\end{array}$ & Estudo transversal \\
\hline $\begin{array}{l}\text { Polejack \& Seidl, } \\
2010\end{array}$ & $\begin{array}{l}\text { Monitoramento e avaliação da adesão ao } \\
\text { tratamento antirretroviral para HIV/AIDS: } \\
\text { desafios e possibilidades }\end{array}$ & $\begin{array}{l}\text { Discutir as possibilidades e desafios no processo de } \\
\text { monitoramento e avaliação da adesão ao tratamento } \\
\text { antirretroviral, bem como as vantagens e desvantagens das } \\
\text { técnicas disponíveis para sua aferição, com destaque para } \\
\text { o trabalho com pessoas de baixa escolaridade e em países } \\
\text { com recursos limitados }\end{array}$ & Revisão sistemática \\
\hline Ruiz et al., 2011 & $\begin{array}{l}\text { Terapia antirretroviral inicial (ARV) em } \\
\text { uma coorte observacional de } 20 \text { anos de } \\
\text { pacientes em seguimento em um centro de } \\
\text { referência na cidade de São Paulo, Brasil }\end{array}$ & $\begin{array}{l}\text { Descrever o tratamento antirretroviral no Centro de } \\
\text { Referência e Treinamento em DST/AIDS em São Paulo. }\end{array}$ & Estudo coorte \\
\hline Santos et al., 2020 & $\begin{array}{l}\text { Perfil epidemiológico dos pacientes } \\
\text { internados por HIV no Brasil }\end{array}$ & $\begin{array}{l}\text { Analisar o perfil epidemiológico dos pacientes internados } \\
\text { por HIV no Brasil, no período de } 2010 \text { a } 2019 .\end{array}$ & $\begin{array}{c}\text { Estudo, retrospectivo, } \\
\text { descritivo, } \\
\text { quantitativo, de base }\end{array}$ \\
\hline
\end{tabular}




\begin{tabular}{|l|l|l|c|}
\hline & \multicolumn{1}{|c|}{ documental. } & \\
\hline Silva et al., 2015 & Fatores associados à não adesão aos & Investigar os fatores associados à não adesão à terapia & Estudo transversal \\
& antirretrovirais em adultos com AIDS nos & antirretroviral de alta potência (TARV) nos primeiros seis & \\
& $\begin{array}{l}\text { seis primeiros meses da terapia em } \\
\text { Salvador, Bahia, Brasil }\end{array}$ & meses de tratamento. & \\
\hline
\end{tabular}

Fonte: Base de dados Scielo e Google Acadêmico (2020).

Embora os números dos tratamentos do HIV/AIDS estão sendo ampliados, ainda é possível observar a importância da detecção precoce, visando não só reduzir a morbimortalidade dos portadores dessa patologia, como também prevenir a transmissão do vírus a outras pessoas. A testagem deve ser ampliada e está indicada para qualquer pessoa sexualmente ativa, em especial aquelas com alto risco de infecção pelo HIV, como as que apresentam sintomas de infecção aguda ou crônica, gestantes, pessoas com IST e aquelas pertencentes a grupos populacionais específicos, como as profissionais do sexo, homens que fazem sexo com homens, transexuais e pessoas que usam álcool e outras drogas (Carvalho, 2021).

Além da ampliação da testagem, o Brasil criou estratégias com ação na distribuição universal e gratuita dos medicamentos antirretrovirais aos portadores do HIV/AIDS, tendo assim efeitos positivos na redução da mortalidade e internações hospitalares, além de redução da incidência das infecções oportunistas e da transmissão vertical do HIV. No entanto, um problema ainda frequente é a não adesão ao tratamento nos diversos contextos socioculturais e econômicos, sendo encontrados como barreiras o medo da descoberta do diagnóstico, o uso abusivo de substâncias psicoativas, o esquecimento e a ausência de compreensão dos benefícios do tratamento. Assim, é fundamental o envolvimento não só do paciente, como também da família, da equipe de saúde e das demais pessoas da rede de apoio, visto que é necessário que haja uma estrutura de seguimento e de apoio psicossocial, buscando auxiliar na adesão às terapias antirretrovirais (Polejack \& Seidl, 2010).

\section{Conclusão}

O tratamento de HIV/AIDS no ano de 2020, portanto, foi mais realizado na região Sudeste, o que pode ser justificado - não só pela maior prevalência - como também pela maior distribuição dos recursos de saúde nessa área de abrangência. Vale ressaltar a importância de se identificarem os fatores que podem reduzir a sobrevida do paciente e com isso poderá contribuir no planejamento das ações de redução da morbimortalidade da doença. A partir dessas informações coletadas, poderão ser planejadas, elaboradas estratégias e aplicação de investimentos direcionados aos pontos essenciais, como a importância do diagnóstico precoce, diagnóstico diferencial, observação nas maneiras de transmissão para melhor intervenção de medidas preventivas, atualização das terapêuticas disponíveis e melhor adesão ao tratamento. Logo, nosso estudo apresenta limitações que são compreendidas pela confiabilidade das informações apresentadas pelo banco de dados do DATASUS e é restringida pelo nível de acurácia e de complexidade das autorizações de internações hospitalares preenchidas.

Para estudos futuros, sugere-se pesquisas a respeito dos fatores que podem aumentar a sobrevida dos pacientes, de modo que se reduzam as taxas de morbimortalidade da patologia. Além de incentivo à adesão ao tratamento, bem como o combate ao preconceito, que ainda assola a sociedade.

\section{Referências}

Batista, R. M., Andrade, S. da S., \& Souza, T. F. M. P. (2021). Prevalência de casos de HIV/AIDS nos últimos 10 anos no Brasil. Research, Society and Development, 10(14), e336101422149. https://doi.org/10.33448/rsd-v10i14.22149

Calado, S. (2015). Análise De Documentos: Método De Recolha E Análise De Dados - PDF Download grátis. Docplayer.com.br. https://docplayer.com.br/12123665-Analise-de-documentos-metodo-de-recolha-e-analise-de-dados.html

Calvetti, P. Ü., Giovelli, G. R. M., Gauer, G. J. C., \& Moraes, J. F. D. de. (2014). Psychosocial factors associated with adherence to treatment and quality of life in people living with HIV/AIDS in Brazil. Fernandes, Brasileiro de Psiquiatria, 63(1), 8-15. https://doi.org/10.1590/0047-2085000000002 
Carvalho, I. P. (2021, March 29). HIV em adolescentes e adultos: recomendações para diagnóstico e tratamento da infecção no Brasil • SciELO em Perspectiva | Press Releases. SciElO Em Perspectiva | Press Releases - Press Releases de Artigos Publicados Pelos Periódicos Da Rede SciELO. https://pressreleases.scielo.org/blog/2021/03/29/hiv-em-adolescentes-e-adultos-recomendacoes-para-diagnostico-e-tratamento-da-infeccao-nobrasil/\#.YaQvJJXMLIX

Chaves, G. C., Hasenclever, L., Osorio-de-Castro, C. G. S., \& Oliveira, M. A. (2015). Strategies for price reduction of HIV medicines under a monopoly situation in Brazil. Revista de Saúde Pública, 49(0). https://doi.org/10.1590/s0034-8910.2015049005459

Costa, F. L., De Jesus Medeiros, R., Medeiros Paungartner, L., Dias Luft, T., Paz Santos, A., Souza Paiva, T., \& Carollo Fernandes, M. T. (2021). Fatores psicossociais envolvidos na adesão ao tratamento do HIV/AIDS em adultos: revisão integrativa da literatura. Saúde Coletiva (Barueri), 11(61), 4990-5005. https://doi.org/10.36489/saudecoletiva.2021v11i61p4990-5005

Coutinho, M. F. C., O’Dwyer, G., \& Frossard, V. (2018). Tratamento antirretroviral: adesão e a influência da depressão em usuários com HIV/Aids atendidos na atenção primária. Saúde Em Debate, 42(116), 148-161. https://doi.org/10.1590/0103-1104201811612

Da Saúde, M. (2020). Procedimentos hospitalares do SUS. http://tabnet.datasus.gov.br/cgi/tabcgi.exe?sih/cnv/qiuf.def

Fernandes, R. A., Carolina, A., Decimoni, T., Ferrarezzo, F., Zillmer, Vinicius Danzmann, \& Tarabay, J. (2020). Tratamento do HIV/AIDS no Brasil: impacto da adesão sobre a utilização de recursos e custos. Jornal Brasileiro de Economia da Saúde (Impr.), 81-87. https://pesquisa.bvsalud.org/controlecancer/resource/pt/biblio-1096413?src=similardocs

Gratão, L. H. A., Nascimento, G. N. L. do, \& Pereira, R. J. (2021). Effects of HAART in the nutritional status of children and adolescents infected by HIV in Brazil: a systematic review. Ciência \& Saúde Coletiva, 26(4), 1346-1354. https://doi.org/10.1590/1413-81232021264.13862018

Greco, D. B. (2016). Trinta anos de enfrentamento à epidemia da Aids no Brasil, 1985-2015. Ciência \& Saúde Coletiva, 21(5), 1553-1564. https://doi.org/10.1590/1413-81232015215.04402016

Martins, R. S. (2020). Adesão ao tratamento antirretroviral para o HIV/AIDS : uma análise dos eventos marcadores associados à adesão em um estudo de coorte no município de Alvorada - RS. Ufrgs.br. https://doi.org/http://hdl.handle.net/10183/213432

Mendicino, C. C. P., Moodie, E. E. M., Guimarães, M. D. C., \& Pádua, C. A. M. de. (2021). Immune recovery after antiretroviral therapy initiation: a challenge for people living with HIV in Brazil. Cadernos de Saúde Pública, 37(9). https://doi.org/10.1590/0102-311x00143520

Monteiro, S. S., Brigeiro, M., Vilella, W. V., Mora, C., \& Parker, R. (2019). Desafios do tratamento como prevenção do HIV no Brasil: uma análise a partir da literatura sobre testagem. Ciência \& Saúde Coletiva, 24(5), 1793-1807. https://doi.org/10.1590/1413-81232018245.16512017

L9313. (2021). Planalto.gov.br website: https//www.planalto.gov.br/ccivil_03/leis/19313.htm

Oliveira, R. da S., Primeira, M. R., Santos, W. M. dos, Paula, C. C. de, \& Padoin, S. M. de M. (2020). Association between social support and adherence to anti-retroviral treatment in people living with HIV. Revista Gaúcha de Enfermagem, 41. https://doi.org/10.1590/1983-1447.2020.20190290

Padoin, S. M. de M., Züge, S. S., Juliane, D. A., Marcelo, R. P., Érika, E. P. dos S., \& Cristiane, C. de P. (2015). Mulheres do Sul Brasil em terapia antirretroviral: perfil e o cotidiano medicamentoso. Epidemiologia E Serviços de Saúde, 24(1), 71-78. https://doi.org/10.5123/s1679-49742015000100008

Passos, S. M. K., \& Souza, L. D. de M. (2015). An evaluation of quality of life and its determinants among people living with HIV/AIDS from Southern Brazil. Cadernos de Saúde Pública, 31(4), 800-814. https://doi.org/10.1590/0102-311x00000514

Pinheiro, P. N. da C., Kendall, B. C., Kerr, L. R. F. S., Pickett, K. M., Luna, I. T., Costa, M. I. F. da, \& Luz, L. F. da C. (2020). The south american context of diagnostic disclosure of adolescents infected by HIV/AIDS: a systematic literature review. Revista Da Associação Médica Brasileira, 66(8), 1139-1145. https://doi.org/10.1590/1806-9282.66.8.1139

Pinto Neto, L. F. da S., Perini, F. de B., Aragón, M. G., Freitas, M. A., \& Miranda, A. E. (2021). Protocolo Brasileiro para Infecções Sexualmente Transmissíveis 2020: infecção pelo HIV em adolescentes e adultos. Epidemiologia E Serviços de Saúde, 30(spe1). https://doi.org/10.1590/s16794974202100013.esp1

Polejack, L., \& Seidl, E. M. F. (2010). Monitoramento e avaliação da adesão ao tratamento antirretroviral para HIV/aids: desafios e possibilidades. Ciência \& Saúde Coletiva, 15(suppl 1), 1201-1208. https://doi.org/10.1590/s1413-81232010000700029

Ruiz, E. A. C., Ramalho, M., Tancredi, M. V., Moatti, J. P., Monteiro, A. L. C., Fonsi, M., \& Chauveau, J. (2011). Initial antiretroviral therapy in a 20-year observational cohort of patients followed at a reference center in the City of São Paulo, Brazil. Revista Brasileira de Epidemiologia, 14(1), 86-97. https://doi.org/10.1590/s1415-790x2011000100008

Santos, A. C. F., Mendes, B. S., Andrade, C. F., Carvalho, M. M. de, Espírito-Santo, L. R., D’Angelis, C. E. M., \& Prince, K. A. de. (2020). Perfil epidemiológico dos pacientes internados por HIV no Brasil. Revista Eletrônica Acervo Saúde, 48, e3243. https://doi.org/10.25248/reas.e3243.2020

Silva, J. A. G., Dourado, I., Brito, A. M. de, \& Silva, C. A. L. da. (2015). Fatores associados à não adesão aos antirretrovirais em adultos com AIDS nos seis primeiros meses da terapia em Salvador, Bahia, Brasil. Cadernos de Saúde Pública, 31(6), 1188-1198. https://doi.org/10.1590/0102-311x00106914 